\title{
AUGMENTATION THERAPY IN UNIPOLAR DEPRESSION WITH SUICIDAL BEHAVIOR WITH LITHIUM (EVIDENCE BASED CASE REPORT)
}

\author{
Syarifah Husna ${ }^{1}$, Yusvick M. Hadin ${ }^{2}$, Adriesti Herdaetha ${ }^{3}$, Setyowati Raharjo ${ }^{4}$
}

Correspondence: cun.milano@yahoo.com

${ }^{1}$ Psychiatric Resident Medical Faculty of Universitas Sebelas Maret Surakarta, ${ }^{2}$ Psychiatry Department Rumah Sakit Dr. Moewardi Surakarta,

${ }^{3,4}$ Psychiatry Department RSJ Dr. Arif Zainudin Surakarta

\section{ABSTRACT}

Background: Depressive disorder is the main cause associated with recurrent morbidity which is often associated with suicides. Treatment of depression with antidepressant and electroconvulsive therapy is commonly used and proven effective. Based on previous study, administration of lithium as an augmentation therapy showed efficacy in suicidal behavior. The role of lithium as first line for bipolar disorder in manic episodes, also be an option in unipolar depression with some conditions such as antidepressant resistence, recurrent depression, maintenance therapy which is respond to electroconvulsive therapy and suicide behavioral.

Method: The authors conducted an extensive search of the published literature using several terms, including major depressive, lithium, antidepressant and antisuicidal effects. Relevant article were retrieved from PubMed, Cochrane, Springer, ScienceDirect.

Conclusion: Lithium given along antidepressants has proven effective in treatment of unipolar depression, preventing relaps and also has anti-suicide effect

Keywords: Depressive, lithium, antidepressant, antisuicidal effects.

\section{Article History:}

Received: March 25, 2020

Accepted: July 20, 2020

Published: September 28, 2020
Cite this as: Husna, S., Hadin, Y.M., Herdaetha, A. \& Raharjo, S. Augmentation therapy in unipolar depression with suicidal behavior with lithium (evidence based case report). Journal of Psychiatry Psychology and Behavioral Research; 2020. 2: 16-18.

\section{INTRODUCTION}

Depressive disorders is the main cause associated with recurrent morbidity which is often associated with suicides. Suicidal behavior must be monitored carefully in every psychiatric patient. Suicide and suicidal behavior cause severe personal, social and economic consequences. It was important to note that certain factors can increase a patient's risk of suicide so that might be affect the treatment plan. Suicidal behavior cannot be predicted for the time and the successfull of suicide. So it can be planned to provide therapy that can eliminate suicidal behavior. Suicide is one of the top 2 causes of global death among all ages. Every year, nearly one million people die of suicide (WHO 2014), including around 58,000 in the European Union and 10,000 in Germany. In individuals aged 15-39 years, suicide is the second leading cause of death after an accident; however, the number of suicide attempts is still unclear. The etiology of suicidal behavior is multi factorial, although one of the most common risk factors is psychiatric disorders. It is estimated that the lifetime risk of suicide often occurs in mood disorders (especially major depression) ranging from 6 to $15 \%$ (WHO 2000). Treatment of patients with suicidal behavior is a challenge. ${ }^{1}$
The role of lithium as well as first-line bipolar disorder in manic episodes can also be an option in unipolar depressed patients with some conditions such as treatment resistant depression, recurrent depression, as well as maintenance therapy in patients who respond to electroconvulsive therapy (ECT) and suicidal behavior. ${ }^{2}$ Although many studies have confirmed lithium efficacy in unipolar depression, Nierenberg et al confirmed that lithium augmentation is no more effective than placebo in some patients resistant to antidepressants and lithium have little effectiveness in unipolar depression. ${ }^{3}$

Based on previous research said that lithium administration as an augmentation therapy showed efficacy against improvement especially in treatment-resistant depression, as well as acute depression conditions although there were still many studies that showed different results and larger studies were needed in the future so the authors were interested in knowing the role of lithium as additional therapy in unipolar depressed patients with suicidal behavior.

\section{CASE REPORT}

The patient is a 31-years-old male, elementary school graduated and a merchant. Patient had enough social and 
economic background for daily life. He was the first of three siblings, there was no previous medical history, and no family had a mental disorder. He was married and has two daughters. In the first day, the patient did not answer the examiner at all, he wanted to talk to his wife only. After the first ECT, he still did not answer the examiner, after the last ECT he was able to talked about his problems and complaints with slow volume and intonation but understandable. He felt confusion for 3 weeks and too difficult to expressed what he felt. Patient seems more silent, did not want to left the house and tended to confine himself in the room. He look so sad and cried but did not say the reason. Before that, he was angry and screamed loudly, and also apologized to everyone. The patient said that he was useless to his parents and always blames himself. 1 week before entering the hospital, he did not want to ate and drank, did not want to do any activities. The patient stopped working and did not want to do anything for 3 weeks. The patient said he heard a voice that said he was useless.

Patient is also very difficult to concentrate and difficult to start slept, sometimes could fall asleep but when he woke up at night, he was cried and blame himself for what happened with his parent. 1 week before entering hospital, patient attempted suicide with a clothesline but were not successful because of patient's wife. Patient need to die because he felt useless, and always thought that he was a troublemaker and always did wrong, it was better to ended life. At present, the patient has a slight problem with his brother. Initially the patient's father suffered from lung disease, the patient took his father to see a general practitioner but after so long, there was no improvement. Then his brother brought his father to take some treatment in Jakarta and in the end there was a good change in his father's condition. Then since that incident, the patient is often pensive, silent, sad and crying and blame himself because he cannot help his.

When hospitalized the patient was treated with Sertralin 1 x 50 mg, Quetiapine 1 x $200 \mathrm{mg}$ at night and administered ECT 3 sessions.

\section{METHOD}

The authors conducted an extensive search by scientific journals through trusted and frequently used databases, namely PubMed, Cochrane, Springer, and Science Direct. The keyword used is "lithium" AND "major depressive" AND "antidepressant" AND "antisuicidal effects", with a journal publication filter, the last 10 years, with a randomized clinical trial, systematic review, meta-analysis, human research subjects and English.

\section{RESULT}

Lithium has long been used as a therapy in acute bipolar and manic disorders but very few have examined lithium and its potential in acute depression in unipolar depression. Recent comprehensive studies have shown that lithium can prevent recurrence in unipolar depression. Although many types of drugs can be given to major depression, the overall outcome is usually far from optimal. About $30-50 \%$ of patients with major depression are not enough to respond to first-line therapy. Lithium has been used as an augmentation for more than 25 years. The first study conducted in major depressed patients by Montigny et al. reported adding lithium responds quickly to patients who did not respond to tricyclic antidepressants for 3 weeks. ${ }^{4}$
Subsequent randomized controlled trials have been confirmed by Montigny given additional lithium with an average of 30 days with doses between $300-1,500 \mathrm{mg} /$ day versus antidepressants (including SSRIs, tricyclics and tetracyclic doses not reported in this study) there is a greater response to lithium. A recent meta-analysis of 10 randomized, double-blind, plcebo-controlled trials of depressed patients showed that lithium had a significant positive effect compared with placebo. But 5 studies did not show any significant differences. The things that caused the negative discovery were due to use of small lithium doses, short duration of treatment. Previous studies have shown that only lithium doses above 600 $\mathrm{mg} /$ day and with a minimum duration of 7 days are useful in augmentation therapy. ${ }^{4}$

A double-blind placebo-controlled RCT with 30 patients with refractory unipolar depression given lithium and placebo for four months reported one patient attempting suicide in the placebo group and not in the lithium group. Several other RCTs reported suicide attempts in the placebo group and there were no attempts at suicide in the lithium group but there were also RCTs reporting the same between lithium and placebo that had attempted suicide. ${ }^{1}$

A meta-analysis study of several studies of bipolar and unipolar depression patients using lithium and other drugs (anticonvulsants, antipsychotics) showed lower suicide in lithium group (either bipolar or unipolar) with lithium levels reached $0.645-0.811 \mathrm{mEq} / \mathrm{L}^{5}$

\section{DISCUSSION}

It has been explained that suicide is the biggest cause of death globally. Depression disorders are the main cause associated with recurrent morbidity which is often associated with suicides. In depression there is an imbalance or decrease in neurotransmitters. It is clear the efficacy of antidepressant treatment in improving symptoms of depression. However, to reduce the risk of suicide in depressed patients, lithium can be given in accordance with previous studies that confirm the benefits of lithium not only as a first-line bipolar patient but can also be given to unipolar depressed patients with certain conditions.

The unique anti-suicide effect in the mood stabilizer is only found in lithium. The mechanism which is not clearly known regarding the effects of lithium can be explained by several hypotheses such as serotonin (5-HT) and endocrine system activity and modulation. According to Montigny et al., Lithium augmentation can increase 5-HT, possibly through the synergy of action of lithium and antidepressants in the serotonin pathway in the brain. Others have argued that the effect of lithium on 5-HT relates to the activity of agonists partial on 5HT1B autoreceptors to modulate activity at these receptors. The administration of lithium causes increased serotonin turnover in several major regions in the brain to mediate emotional stimulation, cognitive function and impulse control which ultimately reduce suicidal-related behavior. Low serotonergic function is associated with suicide attempts in depressed patients. With compensation for decreased serotonergic activity, lithium may have an impact in reducing the risk of suicide. ${ }^{6}$

There is strong evidence from meta-analysis studies related to lithium administration that it can reduce suicidal behavior in both bipolar and unipolar depression. Comparison with carbamazepin shows a smaller clinical effect compared to lithium. Other meta-analyzes have concluded that it shows 
antisuicidal specific effects and supports the notion of longterm lithium treatment as a strategy for suicide prevention. ${ }^{6}$ There is some debate in patients who get SSRI antidepressants because they have similar anti-suicide effects as lithium. But it is not a big problem, overall serotonergic activity of SSRIs is very different that lithium and the behavioral effects of lithium and SSRIs cannot be compared. ${ }^{6}$

A pharmacogenetic study investigated the association of GSK3Beta -50T / C single nucleotide polymorphism (SNP) and response to lithium augmentation in 81 unipolar depressed patients. Inhibition of GSK3 Beta - intracellular kinase, highly expressed in the brain - is related to neurotropic and neuroprotective effects. What's interesting is that lithium is directly and significantly inhibits GSK3Beta. 50T / C localized single nucleotide polymorphism (SNP) in the GSK3Beta region has been found previously in bipolar patients who respond to lithium prophylaxis. This shows the role of 50T / C-SNP from the GSK3Beta gene against lithium augmentation response in depressed patients who do not respond adequately with antidepressant monotherapy. However, confirmation is still needed with a larger sample. ${ }^{2}$

ECT is an effective therapy for severe depression, resistant to treatment but the incidence of relapse is also high in patients who get ECT. The first prospective study of 1-year lithium advanced therapy in patients who received ECT was very significant in reducing relapse and was effective in preventing recurrence (new episodes) in unipolar depression. ${ }^{7}$ In several RCT studies comparing antidepressant administration with the administration of advanced post-ECT lithium therapy showed that the group receiving lithium could maintain remission. The NIMH (National Institute of Mental Health) recommends a minimum of 6 months of continued administration of lithium. Lithium can be given along with tricyclics and SSRIs. ${ }^{8}$

The relationship between depression and suicide is so strong that antidepressants are needed to help reduce the risk of suicide, but it is not recommended for bipolar depression because it is unsafe and ineffective for long-term use. The effectiveness of lithium in the prevention of suicide is related to impulsive and aggressive reduction associated with depresso or dysforic-agitation associated with suicidal behavior. ${ }^{5}$

Implementation in this case, there was clinical improvement in depression and decrease suicidal behavior after lithium administrated and there was no side effects have been seen in this patient.

\section{CONCLUSION}

Suicide risk was decreased in patients treated with lithium and antidepressant augmentation which is lithium is an augmentation therapy was found as the best evidence in the treatment of depressed patients who do not respond to antidepressants and suicidal behavior.

\section{Conflict of interest}

No conflict of interest in this study.

\section{References}

1. Lewitzka, U. et al.: The suicide prevention effect of lithium: more than 20 years of evidence - a narrative review', International Journal of Bipolar Disorders (2015).

2. Bschor, T.: Lithium in the Treatment of Major Depressive Disorder (2014)
3. Nelson, J. C. et al.: A systematic review and meta-analysis of lithium augmentation of tricyclic and second generation antidepressants in major depression. journal of affective disorders. Elsevier (2014)

4. Bauer, M.: Lithium's Emerging Role in the Treatment of Refractory Major Depressive Episodes. pp. 36-42 (2010)

5. Tondo L. And Baldessarini R.J.: Antisuicidal effects in mood disorder: are they unique to lithium? Pharmacopsychiatry (2018)

6. Lauterbach, E. et al.: Adjunctive lithium treatment in the prevention of suicidal behaviour in depressive disorders, a randomized. pp. 469-479 (2008)

7. Rasmussen, K. G.: Lithium for Post-Electroconvulsive Therapy Depressive Relapse Prevention A Consideration of the Evidence. pp 87-90 (2015)

8. Saleh, M., et al.: Lithium in the episode and suicide prophylaxis and in augmenting strategies in patients with unipolar depression. International Journal of Bipolar Disorders. Springer Berlin Heidelberg (2017) 